\title{
A Review of Quality of Life Measures in Dry Eye Questionnaires
}

Joseph R. Grubbs Jr, MPH${ }^{*}$, , Sue Tolleson-Rinehart, $\mathrm{PhD}^{\star}, \dagger$, Kyle Huynh ${ }^{\ddagger}$, , and Richard M. Davis, MDI

*University of North Carolina School of Medicine, Chapel Hill, NC

†University of North Carolina Gillings School of Global Public Health, Chapel Hill, NC

‡University of North Carolina, Chapel Hill, NC

§School of Medicine and Health Sciences, George Washington University, Washington, DC

IDepartment of Ophthalmology, University of North Carolina, Chapel Hill, NC

\section{Abstract}

Purpose-Dry eye disease (DED) is an ocular disease that affects 5\% to $17 \%$ of the US population. Because of the negative effects of DED on patients' quality of life (QOL), diseasespecific questionnaires that assess QOL in patients with dry eyes are essential in the monitoring and management of this chronic ocular condition. This review provides clinicians and researchers with a summary of the current questionnaires available for assessing QOL in patients with dry eyes.

Methods-A systematic review of the literature was performed in March 2013.

Results-There are only 2 validated, reliable dry eye questionnaires with QOL measures currently available for clinic use: the Ocular Surface Disease Index (OSDI) and the Impact of Dry Eye on Everyday Life questionnaire (IDEEL). Multiple other dry eye questionnaires assess some degree of QOL, but they have either not been tested for validity and reliability or are limited in QOL measures they assess.

Conclusions-The OSDI and IDEEL are validated, reliable disease-specific questionnaires that assess QOL measures in patients with DED. Because of its extensive development process and multiple QOL measures, the IDEEL offers a more thorough assessment of the effect of DED on QOL for clinical trials, whereas the OSDI may be the more convenient option for clinical use as a result of its shorter completion time. Other questionnaires used to assess QOL in DED (eg, 25item National Eye Institute Visual Function Questionnaire and Texas Eye Research and Technology Center DEQ) are fairly limited in this assessment. Finally, because of the negative effects of DED on QOL, this review emphasizes the importance of including QOL measures in future questionnaires for the monitoring and management of DED.

Copyright (C) 2013 by Lippincott Williams \& Wilkins

Reprints: Joseph Grubbs, Jr, 1006 Kingswood Dr, Apt J, Chapel Hill, NC 27517 (joseph_grubbs@med.unc.edu).

The authors have no funding or conflicts of interest to disclose. 


\section{Keywords}

quality of life; dry eye; questionnaires

Dry eye disease (DED) is a common ocular disease characterized by eye pain, burning, foreign body sensation, and ocular surface irritation. ${ }^{1,2}$ This disease affects $5 \%$ to $17 \%$ of the US population, and the evidence that DED can greatly diminish quality of life (QOL) in the affected population is steadily increasing. ${ }^{2-6}$ Recent QOL studies of DED show that moderate-to-severe dry eye can cause a decrease in the QOL comparable with that in dialysis, severe angina, and disabling hip fractures. ${ }^{6,7}$

Several clinical dry eye tests are readily available to help monitor the severity of DED, including fluorescein staining, tear break-up time, lissamine green staining, Schirmer test type 1 , Schirmer test type 2 , and many other clinical measures. ${ }^{2,8,9}$ Unfortunately, these tests correlate poorly with patient symptoms and QOL measures in patients with dry eyes. ${ }^{2}$ Nevertheless, these objective tests, including a novel test for measuring tear film osmolarity, are vitally important in the screening and diagnosis of DED because multiple studies have shown that many patients with DED are largely asymptomatic and would not be diagnosed by a symptom-based screening method alone. ${ }^{10,11}$ At the same time, the limited correlation between signs and symptoms makes assessing QOL of DED patients through patientreported questionnaires an important component of DED monitoring and management because many patients are more symptomatic and have a worse QOL than their clinical signs alone may suggest. ${ }^{1,12}$ In addition, the Federal Food and Drug Administration (FDA) has increased their emphasis on properly developing and using patient-reported outcomes (PROs), such as QOL measures, in clinical drug trials to determine treatment efficacy. ${ }^{13}$ As a result of this increased emphasis on proper PRO development and the weak correlation of clinical tests to patient-reported symptoms in DED, it is essential that ophthalmologists be aware of PRO questionnaires currently available for assessing QOL in patients with dry eyes.

The International Dry Eye Workshop highlighted several of these questionnaires in its 2007 epidemiology report on DED; however, it only provided brief descriptions of the questionnaires available for assessing QOL in patients with dry eyes. ${ }^{8}$ Subsequently, in 2010, Alcon, inc. (Ft. Worth, TX) funded and coordinated a systematic review of PRO instruments available for use in clinical trials involving DED. The authors based their review on the 2007 Dry Eye Workshop report and their own 2010 literature search. This 2010 review highlights several dry eye questionnaires that assess QOL and provide detailed descriptions of and recommendations for these PRO instruments. ${ }^{12}$ Yet, no other systematic reviews have been performed since that time to confirm the results of this study even though more work on dry eye questionnaires has been published since their literature review. $4,14,15$ Therefore, the current review article seeks to provide clinicians and researchers with an update on the dry eye questionnaires currently available to assess QOL in patients with DED. 


\section{METHODS}

A systematic review of the literature was performed at the University of North Carolina in March 2013, to determine the validated, reliable dry eye questionnaires with QOL measures that are currently available for clinicians and researchers to use. Because of the FDA's recent emphasis on proper PRO development, a "validated, reliable dry eye questionnaire with QOL measures" was defined as a questionnaire that is disease specific, has undergone validity and reliability testing (including test-retest reliability) in a DED population, includes QOL measures, and has been developed and tested in the English language. This review was not externally funded.

\section{RESULTS}

Two validated, reliable dry eye questionnaires that include QOL measures are currently available for clinic use: the Ocular Surface Disease Index (OSDI) and the Impact of Dry Eye on Everyday Life questionnaire (IDEEL). 2,9,14,16 These 2 questionnaires are summarized in detail below. This systematic review also uncovered 7 other questionnaires used in DED populations that assess some degree of QOL, but these questionnaires do not meet the aforementioned criteria for being a validated, reliable dry eye questionnaire with QOL measures. Because these questionnaires may still prove useful as a supplement to the OSDI and IDEEL, however, a brief overview of these questionnaires has been provided as well.

\section{The OSDI}

Description-The OSDI is a 12-item questionnaire that assesses both dry eye symptoms and their effects on vision-related functioning over the past week. It contains 3 subsections including vision-related function, ocular symptoms, and environmental triggers. $1,9,12$ Patients are asked about the frequency of occurrence of various symptoms and difficulty encountered with vision-related activities and for their response on a 0 to 4 scale that ranges from "none of the time" to "all of the time." The final score is calculated by multiplying the sum of all the scores by 25 and then dividing the total by the number of questions answered. ${ }^{9}$ Scores range from 0 to 100 with 0 to 12 representing normal, 13 to 22 mild DED, 23 to 32 moderate DED, and $\bullet 33$ severe DED., ${ }^{9,17}$

Validity and Reliability Testing-The OSDI has undergone substantial validity and reliability testing since its initial development. ${ }^{9,12}$ The OSDI as a whole and its 3 subscales individually are all internally consistent and display good-to-excellent test-retest reliability. ${ }^{2,9}$ Although the questionnaire is only weakly correlated with clinical dry eye tests, it is strongly correlated with several other dry eye questionnaires and has moderate correlations with artificial tear usage. ${ }^{9}$ The scale is capable of accurately discriminating between normal, mild-to-moderate, and severe DED. ${ }^{12}$ In addition, a minimal clinically important difference has been determined for mild-to-moderate disease (4.5-7.3) and severe disease (7.3-13.4). . $^{2,12,17}$

QOL Measures-The main QOL measures in the OSDI are in its vision-related function subscale. This subscale consists of 6 questions that assess the frequency of problems with vision and vision-related activities such as reading, watching television, driving, and using a 
computer or an automated teller machine. ${ }^{8,16}$ These 6 questions are included in the overall OSDI score and can be computed into their own vision-related function subscale score as well. ${ }^{9}$

Limitations-One limitation of the OSDI is that it only includes some dry eye symptoms such as grittiness, sensitivity to light, and pain but does not include other symptoms such as foreign body sensation and tearing. ${ }^{14,16}$ It also only discusses some of the effects of DED on vision-related functioning; therefore, it may not capture the entire effect of DED on a patient's daily living. ${ }^{14}$ In addition, OSDI responses are limited to measures of frequency (rather than to frequency and severity). ${ }^{18} \mathrm{~A}$ second limitation of the OSDI is the effort required to complete and score the scale, which may consume valuable clinic time in a practice. $^{19}$

Overall Utility-Overall, the OSDI is a valid, reliable dry eye questionnaire that at least partially captures the effects of DED on QOL. Even with its limitations, the OSDI has proven to be a valuable PRO measure in clinical trials and in ophthalmology clinics. ${ }^{1,9,12}$

\section{The IDEEL}

Description-The IDEEL is a 3-module questionnaire with 57 questions, and it assesses dry eye symptoms, the effect of DED on QOL, and treatment satisfaction over the past 2 weeks. ${ }^{8,14,15}$ The 3 modules consist of 6 scales that are outlined below:

- Dry eye impact on daily life: activity limitations, work limitations, and emotional impact.

- Dry eye treatment satisfaction: satisfaction with treatment and treatment-related inconvenience.

- Dry eye symptom (-related) bother (which is its own scale). ${ }^{2,14}$

- Each module is scored from 0 to 100 . Higher scores on the impact on daily life module and treatment satisfaction module indicate a better QOL and higher treatment satisfaction. Higher scores on the symptom bother module indicate more bothersome dry eye symptoms. ${ }^{14}$ For the symptom bother module, $40.0(67.5)$ is the average score of patients with mild DED, 50.6 (6 11.0) for patients with moderate DED, and 64.3 (6 8.0) for patients with severe DED. ${ }^{12}$

Validity and Reliability-The IDEEL meets the new FDA PRO instrument development guidelines and has undergone significant validity and reliability testing. ${ }^{14}$ In addition, content validity has been confirmed by strong evidence of item saturation (ie, multiple interviews confirmed that the questionnaire included the relevant questions needed to achieve its assessment objective). ${ }^{12,14}$ The questionnaire is internally consistent and has good test-retest reliability. ${ }^{12}$ The IDEEL weakly correlates with clinical dry eye tests and generic QOL questionnaires (Short Form-36 and EuroQoL-5D). In addition, its modules have mild-to-strong correlations with the dry eye questionnaire. ${ }^{5,14}$ The Symptom Bother and Impact on Daily Life modules are able to discriminate among mild, moderate, and 
severe DED. ${ }^{4,12}$ In addition, a minimal clinically important difference of 12 points has been determined for the Symptom Bother module. ${ }^{20}$

QOL Measures-The IDEEL has an entire module dedicated to assessing the effects of DED on a patient's QOL. The Impact on Daily Life module consists of 31 questions that assess the effects of DED on a patient's emotions (eg, irritability, sadness), activities (eg, driving, doing close work), and work (eg, difficulty concentrating, feeling distracted). ${ }^{14}$ These 31 questions ultimately produce a score for the entire Impact of Daily Life module. ${ }^{14}$ Finally, the IDEEL's Impact on Daily Life and Symptom Bother modules are better able to discriminate among mild, moderate, and severe DED than can other QOL questionnaires such as the Short Form-36 and EuroQoL-5D. ${ }^{5}$

Limitations-The primary limitation of the IDEEL is that it takes approximately 30 minutes to complete the entire questionnaire. ${ }^{2}$ This limitation is partially offset by the fact that individual modules can be used in practice rather than the entire questionnaire. ${ }^{1}$ One final limitation of the IDEEL is that it must be purchased for use. ${ }^{1}$

Overall Utility-The IDEEL is a valid, reliable dry eye questionnaire that does an excellent job of assessing the effects of DED on QOL. It contains the most QOL measures of any current dry eye questionnaire. Its overall utility is somewhat uncertain because of its recent development and thus limited use in recent clinical trials, but it holds promise at being an important PRO questionnaire for measuring QOL in patients with DED. ${ }^{1,8}$ In addition to its cost, the major limitation of this questionnaire's use in practice may be that it is time consuming. This limitation, however, may not be as restricting in clinical trials.

\section{Other Questionnaires}

Although the OSDI and IDEEL are the only 2 disease-specific, validated, reliable questionnaires that assess the effect of DED on QOL, other questionnaires have been used to assess QOL measures in patients with DED. The 25-item National Eye Institute Visual Function Questionnaire (NEI-VFQ-25) assesses the effect of various eye diseases on QOL. ${ }^{1,21}$ It is not disease specific, but it has been tested in patients with dry eyes, and it displays a modest to strong correlation with the OSDI. $1,6,7,12,18$ Of particular note, the NEIVFQ-25's ocular pain subscale had the strongest overall correlation with the OSDI leading some researchers to suggest that patients with low ocular pain scores on the NEI-VFQ-25 should undergo further dry eye testing. ${ }^{18,22}$ Limitations of the NEI-VFQ-25 for assessing QOL in patients with dry eyes are that it is not disease specific, needs further validity and reliability testing in a dry eye population, lacks a specified recall period, and requires 10 minutes to administer the questionnaire. ${ }^{1,12}$

The original DEQ and the contact lens dry eye questionnaire (includes extra questions for contact lens wearers) include questions about DED symptoms affecting daily activities, but overall, both questionnaires (and subsequent variations of these questionnaires) include very few QOL measures and need reliability testing. ${ }^{12,23,24}$ The Texas Eye Research and Technology Center DEQ is a 33-item questionnaire based on the original dry eye questionnaire that adds several components to the original dry eye questionnaire, including 2 
questions on the disease effect on QOL. ${ }^{12,25}$ The questionnaire has undergone some validity testing and can discriminate between normal patients and patients with moderate dry eyes. Limitations of the questionnaire for measuring QOL in patients with dry eyes include the need for further test-retest reliability testing. In addition, only a small portion of the questionnaire is dedicated to QOL measures. ${ }^{25}$ Thus, these 3 questionnaires are fairly limited in their ability to assess the effect of DED on QOL.

Several other dry eye questionnaires reported in the literature include QOL measures. First, an 11-Question Dry Eye Syndrome Questionnaire including DED-specific QOL questions was used among participants in the Women's Health Study and Physicians' Health Study to determine the effects of dry eye on daily living. ${ }^{3}$ Second, a Single Item Score dry eye questionnaire that attempts to quantify dryness-caused discomfort was used in a recent study to determine whether or not patients with dry eyes were currently symptomatic; this questionnaire showed strong correlations with the OSDI and some clinical tests. ${ }^{12,26}$ Both these questionnaires may assess some QOL measures in patients with dry eyes but are poorly described in the literature and need to undergo substantially more validity and reliability testing before being recommended for clinical use. ${ }^{3,12}$

One final DED questionnaire that measures QOL is the Ocular Surface Disease questionnaire (OSD). ${ }^{12}$ This includes 4 modules, one of which is QOL (OSD-QOL). The OSD-QOL module has been clinically validated and displays a low-to-excellent internal consistency and test-retest reliability. ${ }^{12}$ Its main limitations include the need for further validity and reliability testing of the other modules (medical management/history, symptoms, and treatment satisfaction), lack of data about testing and use in the English language, and no recall period provided in the questionnaire. ${ }^{12}$ Once these limitations are addressed, the OSD may serve as a valuable tool in the clinic. ${ }^{12}$

\section{CONCLUSIONS}

This review provides a brief analysis of the dry eye questionnaires currently available to assess the vision-related QOL in patients with DED and should assist clinicians and researchers alike in determining which questionnaire is the best for their individual situation such as a clinical practice or a clinical trial. To summarize, there are 2 validated, reliable disease-specific questionnaires that assess QOL measures in patients with DED: the OSDI and IDEEL. The IDEEL underwent an extensive development process, contains the most QOL measures of these 2 questionnaires, and may offer the best assessment of the effect of DED on QOL for clinical trials. The OSDI, however, can be completed much more quickly than the IDEEL can, is free for use, and thus may be the more convenient option for use in the clinic. Other questionnaires (eg, NEI-VFQ-25 and Texas Eye Research and Technology Center DEQ) can be used to assess QOL in patients with dry eyes but are extremely limited in this assessment because of a lack of QOL measures or a lack of validity and reliability testing. This review highlights the need for further testing of these dry eye questionnaires.

Finally, multiple studies continue to show DED's deleterious effects on patients' QOL, and PRO questionnaires remain one of the best ways to measure this disease effect on a patient's daily living. ${ }^{12}$ This review emphasizes the importance of including such QOL measures in 
future PRO questionnaires for DED. An increased emphasis on QOL in dry eye questionnaires will not only provide valuable outcome data for clinical trials but will also assist in the clinical treatment and management of DED.

\section{References}

1. Nichols KK. Patient-reported symptoms in dry dye disease. Ocul Surf. 2006; 4:137-145. [PubMed: 16900269]

2. Friedman NJ. Impact of dry eye disease and treatment on quality of life. Curr Opin Ophthalmol. 2010; 21:310-316. [PubMed: 20467319]

3. Miljanović B, Dana R, Sullivan DA, et al. Impact of dry eye syndrome on vision-related quality of life. Am J Ophthalmol. 2007; 143:409-415. [PubMed: 17317388]

4. Pouyeh B, Viteri E, Feuer W, et al. Impact of ocular surface symptoms on quality of life in a United States veterans affairs population. Am J Ophthalmol. 2012; 153:1061-1066. e3. [PubMed: 22330309]

5. Rajagopalan K, Abetz L, Mertzanis P, et al. Comparing the discriminative validity of two generic and one disease-specific health-related quality of life measures in a sample of patients with dry eye. Value Health. 2005; 8:168-174. [PubMed: 15804325]

6. Schiffman RM, Walt JG, Jacobsen G, et al. Utility assessment among patients with dry eye disease. Ophthalmology. 2003; 110:1412-1419. [PubMed: 12867401]

7. Buchholz P, Steeds CS, Stern LS, et al. Utility assessment to measure the impact of dry eye disease. Ocul Surf. 2006; 4:155-161. [PubMed: 16900272]

8. The epidemiology of dry eye disease: report of the Epidemiology Subcommittee of the International Dry Eye WorkShop (2007). Ocul Surf. 2007; 5:93-107. [PubMed: 17508117]

9. Schiffman RM, Christianson MD, Jacobsen G, et al. Reliability and validity of the Ocular Surface Disease Index. Arch Ophthalmol. 2000; 118:615-621. [PubMed: 10815152]

10. Sullivan BD, Crews LA, Messmer EM, et al. Correlations between commonly used objective signs and symptoms for the diagnosis of dry eye disease: clinical implications. Acta Ophthalmol. 2012 [epub ahead of print]. 10.1111/aos.12012

11. Viso E, Rodríguez-Ares MT, Abelenda D, et al. Prevalence of asymptomatic and symptomatic meibomian gland dysfunction in the general population of Spain. Invest Ophthalmol Vis Sci. 2012; 53:2601-2606. [PubMed: 22427596]

12. Guillemin I, Begley C, Chalmers R, et al. Appraisal of patient-reported outcome instruments available for randomized clinical trials in dry eye: revisiting the standards. Ocul Surf. 2012; 10:84-99. [PubMed: 22482469]

13. Bottomley A, Jones D, Claassens L. Patient-reported outcomes: assessment and current perspectives of the guidelines of the Food and Drug Administration and the reflection paper of the European Medicines Agency. Eur J Cancer. 2009; 45:347-353. [PubMed: 19013787]

14. Abetz L, Rajagopalan K, Mertzanis P, et al. Development and validation of the impact of dry eye on everyday life (IDEEL) questionnaire, a patient-reported outcomes (PRO) measure for the assessment of the burden of dry eye on patients. Health Qual Life Outcomes. 2011; 9:111. [PubMed: 22152125]

15. Hernández-Molina G, Sánchez-Hernández T. Clinimetric methods in Sjögren’s syndrome. Semin Arthritis Rheum. 2013; 42:627-639. [PubMed: 23352255]

16. Allergan, I. [Accessed March 31, 2013] Ocular Surface Disease Index (OSDI) [The Dry Eye Zone website]. 1995. Available at: http://www.dryeyezone.com/encyclopedia/documents/OSDI.pdf

17. Pflugfelder SC, Baudouin C. Challenges in the clinical measurement of ocular surface disease in glaucoma patients. Clin Ophthalmol. 2011; 5:1575-1583. [PubMed: 22125404]

18. Vitale S, Goodman LA, Reed GF, et al. Comparison of the NEI-VFQ and OSDI questionnaires in patients with Sjögren's syndrome-related dry eye. Health Qual Life Outcomes. 2004; 2:44.

[PubMed: 15341657] 
19. McDonald M, D’Aversa G, Perry HD, et al. Correlating patient-reported response to hydroxypropyl cellulose ophthalmic insert (LACRISERT ${ }^{\circledR}$ ) therapy with clinical outcomes: tools for predicting response. Curr Eye Res. 2010; 35:880-887. [PubMed: 20858108]

20. Fairchild CJ, Chalmers RL, Begley CG. Clinically important difference in dry eye: change in IDEEL-symptom bother. Optom Vis Sci. 2008; 85:699-707. [PubMed: 18677226]

21. RAND. [Accessed March 31, 2013] National Eye Institute Visual Functioning Questionnaire-25 (VFQ-25) [National Eye Institute website]. 2000. Available at: http://www.nei.nih.gov/resources/ visionfunction/vfq_ia.pdf

22. Nichols KK, Mitchell GL, Zadnik K. Performance and repeatability of the NEI-VFQ-25 in patients with dry eye. Cornea. 2002; 21:578-583. [PubMed: 12131034]

23. Begley CG, Chalmers RL, Mitchell GL, et al. Characterization of ocular surface symptoms from optometric practices in North America. Cornea. 2001; 20:610-618. [PubMed: 11473162]

24. [Accessed March 31, 2013] Dry Eye Questionnaire [Tear Film \& Ocular Surface Society web site]. 2002. Available at: http://www.tearfilm.org/dewsreport/pdfs/Questionnaire\%20DEQ $\%$ 20questionnaire\%20(Caffery).pdf

25. Narayanan S, Miller WL, Prager TC, et al. The diagnosis and characteristics of moderate dry eye in non-contact lens wearers. Eye Contact Lens. 2005; 31:96-104. [PubMed: 15894874]

26. Srinivasan S, Joyce E, Jones LW. Tear osmolality and ferning patterns in postmenopausal women. Optom Vis Sci. 2007; 84:588-592. [PubMed: 17632306] 